\title{
(Auto-)immune signature in aplastic anemia
}

\author{
Antonio M. Risitano \\ Hematology, Department of Clinical Medicine and Surgery, Federico II University, Naples, Italy
}

E-mail:amrisita@unina.it

doi:10.3324/haematol.2018.190884

A cquired idiopathic aplastic anemia (IAA) is a rare hematologic disorder characterized by the failure of hematopoiesis secondary to an immune-mediated damage of the bone marrow. IAA is bona fide considered an auto-immune disease with a T-cell-mediated pathophysiology. ${ }^{1}$ In this issue of the Journal, Giudice et al. ${ }^{2}$ describe the oligoclonal pattern of effector memory $\mathrm{CD}^{+} \mathrm{CD}^{+} 7^{+} \mathrm{T}$ cells in IAA patients by using a combined deep sequencing and flow cytometry approach. Indeed, Giudice et al. show that clonally expanded T-cell populations are frequently detectable even within the effector memory compartment, and that they tend to correlate with disease activity. Thus, the characterization of the T-cell receptor (TCR) repertoire by high-resolution techniques may play a role in confirming the diagnosis of immune-mediated IAA and to monitor affected patients during their disease course.

There is widespread clinical and experimental evidence to support the autoimmune pathophysiology of IAA. ${ }^{1}$ The most striking is that patients with IAA may respond to $\mathrm{T}$ cell-targeted immunosuppressive therapies (IST), with rates of hematologic responses ranging between $50 \%$ and $70 \%{ }^{3}$ Almost four decades of investigations have provided us with a plethora of experimental data corroborating the hypothesis of an immune-mediated pathophysiology. Increased circulating activated $T$ cells were described in IAA patients in the '80s." These T cells may suppress hematopoiesis through the secretion of different inflammatory cytokines ${ }^{5,6}$ and/or via cell-mediated direct killing. Among the different inhibitory cytokines, interferon- $\gamma$ (IFN- $\gamma$ ) plays a major role in suppressing human hematopoietic stem cells (HSC) in vivo, as suggested by in vitro inhibition of cell cycle progression and induction of apoptosis of hematopoietic progenitors. ${ }^{7}$ More recently, it has been suggested that IFN- $\gamma$ may also exert its inhibitory effect on HSC impairing the homeostatic survival signal delivered by thrombopoietin through its cognate receptor c-MPL. ${ }^{8}$ This inhibitory milieu is generated by immune cells, and mostly by $\mathrm{T}$ cells that become activated and proliferate in response to an antigen-driven stimulation. While the search for these putative antigens has remained unsuccessful, the demonstration of clonal expansion of $\mathrm{T}$-cell populations identified by their TCR has been considered robust proof of a T-cell-mediated pathophysiology in IAA., ${ }^{910}$

Our growing understanding of the immune system and the availability of powerful novel techniques has nurtured continuous research in the field of IAA. On the one hand, investigators have tried to further dissect the abnormalities of the immune system in patients suffering from IAA. Indeed, looking at specific functional T-cell subsets, recurrent immune derangements have been found, such as increased T-helper type 17 cells (Th17) ${ }^{11}$ and reduced regulatory $T$ cells (Treg). ${ }^{12,13}$ However, irrespective of the deep phenotyping of $\mathrm{CD}^{+} \mathrm{T}$ cells [i.e. by multiparameter mass cytometry, termed cytometry by time-of-flight (CyTOF)], ${ }^{14}$ only limited data are available about the characterization of specific functional CD8 ${ }^{+} \mathrm{T}$-cell subsets. On the other hand, novel techniques of deep DNA sequencing have become available, and their application in IAA has led to the description of somatic mutations in myeloid cells, ${ }^{15}$ with the subsequent ongoing debate about their actual meaning, ${ }^{16}$ but also to high throughput TCR analysis. ${ }^{17}$ In their study, Giudice et al. specifically investigated the compartment of effector memory $T$ cells $\left(T_{\mathrm{EM}}\right)$ in IAA patients, looking for possible clonality as assessed by flow cytometry analysis of $\mathrm{V} \beta$ usage and sequencing of the hypervariable complementary determining region 3 (CDR3) of the TCR.

In agreement with previous reports, ${ }^{1,10}$ Giudice et al. confirm that AA patients often exhibit a skewed usage of $\mathrm{V} \beta$ families, usually within the CD8 ${ }^{+} \mathrm{T}$-cell compartment; these oligoclonal expansions are more frequent in patients with increased percentage of $\mathrm{T}_{\mathrm{EM}}$ (as defined by co-expression of CD8 and CD57), which are found in approximately $70 \%$ of AA patients. These gross abnormalities of the TCR V $\beta$ usage were dissected at the clonal level by deep sequencing of the TCR, which allows the comparison of more than $10^{7}$ reads corresponding to TCRs harbored by individual $\mathrm{T}$ cells. Indeed, clonal expansions were identified by repetitive use of TCR $\beta$ variable (TRBV) and joint (TRJV) genes (i.e. redundant TRBV/TRBJ combinations), as well as by CDR3 size and DJ length profiles. Immunodominant clones within different T-cell subsets were invariably detected in all AA patients with increased $\mathrm{CD} 8^{+} \mathrm{CD}^{+} 7^{+} \mathrm{T}_{\mathrm{EM}}$; however, the actual magnitude of these clonal expansions was extremely heterogeneous in the different T-cell subsets. Indeed, these clones remain minimally expanded (approx. 3\%) within the $\mathrm{CD}^{+}$compartment, while they become largely dominant (approx. 18\%) in the CD8 ${ }^{+}$compartment; the expansion appears even larger when the $\mathrm{CD} 8^{+} \mathrm{CD} 57^{+} \mathrm{T}_{\mathrm{EM}}$ is analyzed. This difference in behavior of TCR heterogeneity in different T-cell subsets of AA patients was further confirmed by analysis of Simpson's diversity score, which shows how TCR diversity decreased from $\mathrm{CD}^{+}$to $\mathrm{CD}^{+} \mathrm{T}$ cells, and even more from both $\mathrm{CD}^{+}$and $\mathrm{CD}^{+} \mathrm{T}$ cells to $\mathrm{CD}^{+} \mathrm{CD}^{+} 7^{+}$ $\mathrm{T}_{\mathrm{EM}}$. In summary, the work performed by Giudice et al. confirms that clonal $\mathrm{CD}^{+} \mathrm{T}$-cell expansions are common in $\mathrm{AA}$ patients, in agreement with the well-established T-cell-mediated immune pathophysiology of AA. ${ }^{1}$ But for the first time, here the Authors provide evidence that the clonal expansions are not limited to the $\mathrm{CD}^{+}$effector $\mathrm{T}$ cells, since they can be found even in the $\mathrm{T}_{\mathrm{EM}}$ compartment. Very interestingly, with the caveat of a limited sample size, Giudice et al. show that abnormalities of the $\mathrm{T}_{\mathrm{EM}}$ compartment (i.e. increased $\mathrm{T}_{\mathrm{EM}}$, with possible clonal expansions) may be associated with a dismal outcome in AA, due to refractory or relapsed disease. This is further supported by the observation of concordance between the expansion of the immunodominant $\mathrm{CD}^{+} \mathrm{CD}^{+} 7^{+} \mathrm{T}_{\mathrm{EM}}$ clone and disease activity (sim- 
ilar to what has already been shown for expansion within the bulk $\mathrm{CD}^{+}{ }^{+}$compartment $^{10}$ ).

Memory is the hallmark of adaptive immunity; indeed, antigen-driven clonal expansion of effector $\mathrm{T}$ cells may generate antigen-specific lymphocytes that may persist life long (reviewed by Sallusto et al. ${ }^{18}$ ). These cells, which are known as memory cells, confer immediate and effective protection against pathogens upon antigen rechallenging; indeed, memory immune cells are selected for their higher affinity for cognate antigens, and rapidly activate from their resting state after antigen stimulation. ${ }^{18}$ Among $T$ cells, three subtypes of memory cells have been described: $:^{18}$ i) $\mathrm{T}_{\mathrm{EM}}$, that carry the protective memory because of their ability to deliver effector functions; ii) central memory $\mathrm{T}$ cells $\left(\mathrm{T}_{\mathrm{CM}}\right)$, that home to secondary lymphoid organs and exert reactive memory through their cross-differentiation toward $T_{E M}$ and effector $T$ cells ( $T_{\text {EFFF }}$; and iii) memory stem $T$ cells $\left(T_{\mathrm{SCM}}\right)$, which have been described as a less differentiated subset that has better self-renewal and the ability to differentiate into distinct subsets of memory $\mathrm{T}$ cells. Only limited data are available about memory $\mathrm{T}$ cells in AA. In 2009, Hu et al. reported that $T_{E M}$ and $T_{\text {EMRA }}$ (a further subset of terminally differentiated $T_{E M}$ characterized by CD45RA expression and by better effector function) are increased in $\mathrm{CD} 4^{+}$and CD8 ${ }^{+} \mathrm{T}$-cell subsets in AA patients, while naïve $\mathrm{T}$ cells are decreased. ${ }^{19}$ More recently, the US National Institutes of Health group has described that even $\mathrm{T}_{\mathrm{SCM}}$ are increased in circulating CD8 $8^{+} \mathrm{T}$ cells of AA patients. ${ }^{20}$ However, while these observations depict the broad immune derangement which is expected in an autoimmune disease such as AA, they do not provide any clues about the pathogenic role of these cells. In contrast, the demonstration of clonality within the $T_{\mathrm{EM}}$ compartment shown by Giudice et al. seems pathogenically more relevant, according to the scenario depicted in Figure 1. Indeed, a clonal immune response specific for (or crossreactive with) HSC can be elicited by unknown antigens or triggers, eventually causing some impairment of hematopoiesis. If these clonal $\mathrm{T}_{\mathrm{EFF}}$ do not undergo apoptosis (as usually occurrs in a physiological immune response), they may lead to overt AA. ${ }^{1}$ Given the plasticity of $\mathrm{T}$ cells, some of these clonal, antigen-specific cells may eventually acquire a $T_{E M}$ or a $T_{C M}$ functional phenotype, generating some skewing within the broad $\mathrm{T}_{\mathrm{EM}}$ repertoire, as found by Giudice et al. ${ }^{2}$ The presence of these clonal (possibly HSC-specific) $\mathrm{T}_{\mathrm{EM}}$ would account for continuous damage to the hematopoiesis, since in the presence of persistent antigen spread they may serve as a reservoir for newly-generated $T_{\mathrm{EFF}}$. Thus, irrespective of the regulatory role postulated by Giudice et al., ${ }^{2}$ the presence of clonal $T_{E M}$ would represent the signature of a deeper immune derangement, possibly associated with a dismal clinical outcome.

In conclusion, the availability of high-throughput technologies is providing biomarkers which anticipate future

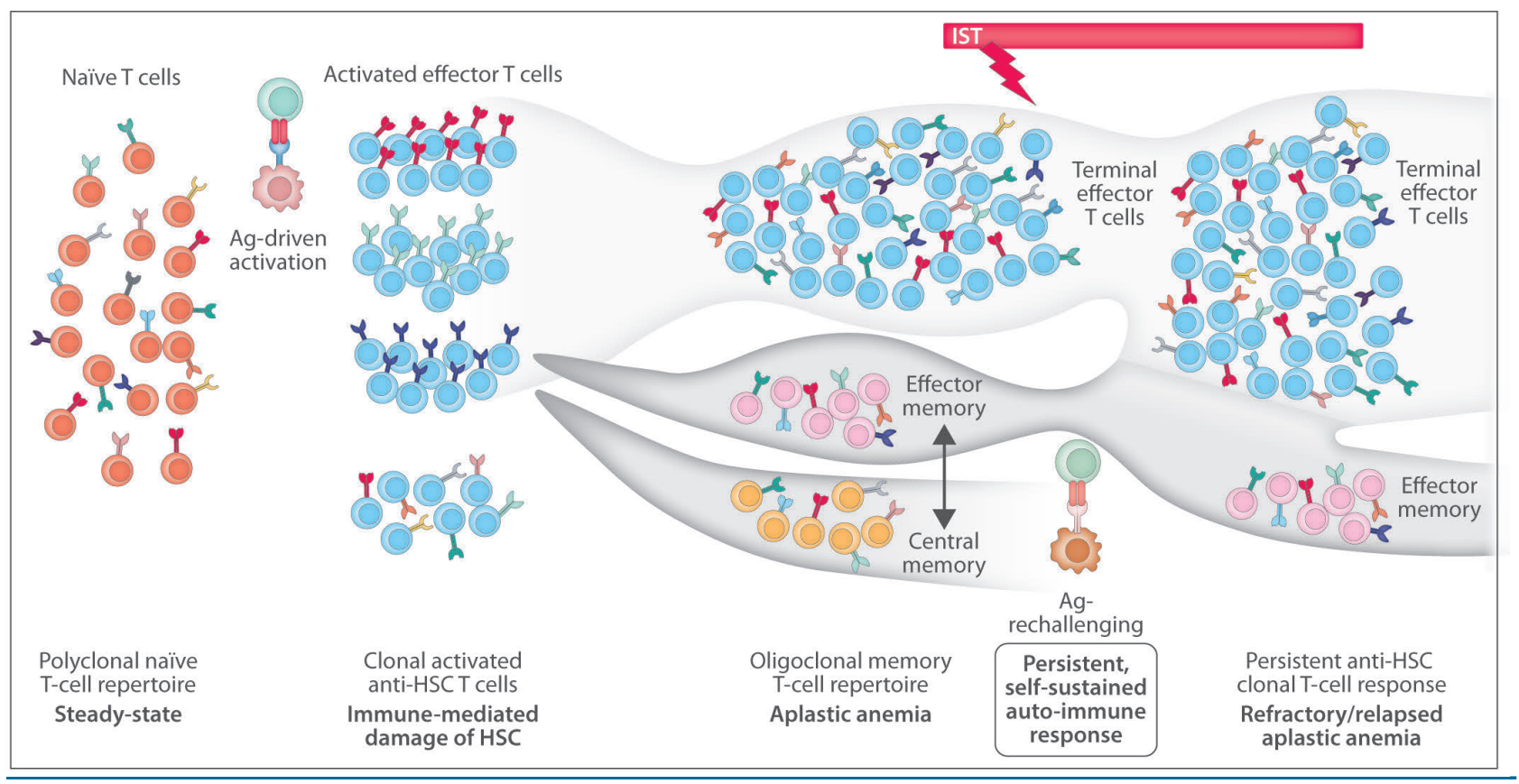

Figure 1. T-cell clonality in aplastic anemia. Unknown antigens and triggers may elicit a clonal immune response specific for (or cross-reactive with) hematopoietic stem cells (HSC). These clonal, activated T cells tend to expand delivering their immune damage over hematopoiesis. At the same time, some of these clonally expanded activated T cells may acquire an effector memory $T$ cell $\left(T_{E M}\right)$ functional phenotype, leading to skewing of the $T_{E M}$ cell repertoire. While activated effector $T$ cells $\left(T_{E F F}\right)$ may undergo anergy or apoptosis (even as a consequence of immunosuppressive therapies), $T_{E M}$ represent a continuous reservoir for HSC-specific $T$ cells, which may exert their effector function upon rechallenging with the antigen. (This is very likely, given the typical antigen spread seen in autoimmune diseases.) Thus, the presence of clonal $T_{E M}$, which likely share the same antigen-specificity with large $T_{E F F}$ clones found in bulk CD $8^{+}$populations, represent a biomarker of a deep-rooted immune derangement, possibly associated with a dismal disease course. 
applications in the management of AA patients. Indeed, deep whole exome sequencing, ${ }^{15} \mathrm{CyTOF}^{14}$ and deep TCR analysis $^{2}$ all help to better describe the pathogenic events underlying bone marrow failure syndromes. Even if none of them translates into immediate therapeutic decisions, they are all useful to confirm the diagnosis, to determine the prognosis and possibly to monitor the clinical course of AA patients. Indeed, this latter application may be useful for early identification of refractory or relapsing patients, paving the way for pre-emptive therapeutic interventions. Moreover, the deep dissection at the clonal and at the functional levels of the immune T-cell compartment (e.g. combining CyTOF and TCR analysis) may also answer some open questions in the field. For example, the differential depletion of some specific T-cell subsets might explain the different outcome seen with different ATG preparations. ${ }^{3}$ These novel technologies may help identify the specific T-cell subsets which are crucial to the pathophysiology of AA (and possibly differentially depleted by distinct ATG brands), possibly driving the development of future targeted therapies.

\section{References}

1. Young NS. Current concepts in the pathophysiology and treatment of aplastic anemia. Hematology Am Soc Hematol Educ Program. 2013;2013:76-81.

2. Giudice V, Feng X, Lin Z, et al. Deep sequencing and flow cytometric characterization of expanded effector memory CD8+CD57+ T cells frequently reveals $\mathrm{T}$-cell receptor Vbeta oligoclonality and CDR3 homology in acquired aplastic anemia. Haematologica. 2018;103(5): 759-769.

3. Scheinberg $\mathrm{P}$, Nunez $\mathrm{O}$, Weinstein $\mathrm{B}$, et al. Horse versus rabbit antithymocyte globulin in acquired aplastic anemia. N Engl J Med. 2011;365(5):430-438.

4. Zoumbos NC, Gascón P, Djeu JY, Trost SR, Young NS. Circulating activated suppressor $\mathrm{T}$ lymphocytes in aplastic anemia. $\mathrm{N}$ Engl J Med. 1985;312(5):257-265.

5. Zoumbos NC, Gascón P, Djeu JY, Young NS. Interferon is a mediator of hematopoietic suppression in aplastic anemia in vitro and possibly in vivo. Proc Natl Acad Sci USA. 1985;82(1):188-192.

6. Sloand E, Kim S, Maciejewski JP, Tisdale J, Follmann D, Young NS
Intracellular interferon-gamma in circulating and marrow $\mathrm{T}$ cells detected by flow cytometry and the response to immunosuppressive therapy in patients with aplastic anemia. Blood. 2002;100(4):11851191.

7. Selleri C, Maciejewski JP, Sato T, Young NS. Interferon-gamma constitutively expressed in the stromal microenvironment of human marrow cultures mediates potent hematopoietic inhibition. Blood: 1996;87(10):4149-4157.

8. Alvarado LJ, Andreoni A, Huntsman HD, et al. Heterodimerization of TPO and IFNy Impairs Human Hematopoietic Stem/Progenitor Cell Signaling and Survival in Chronic Inflammation Blood. 2017;130(Suppl 1):4.

9. Zeng W, Nakao S, Takamatsu H, et al. Characterization of T-cell repertoire of the bone marrow in immune-mediated aplastic anemia: evidence for the involvement of antigen-driven T-cell response in cyclosporine-dependent aplastic anemia. Blood. 1999;93(9):30083016.

10. Risitano AM, Maciejewski JP, Green S, Plasilova M, Zeng W, Young NS. In-vivo dominant immune responses in aplastic anaemia: molecular tracking of putatively pathogenetic T-cell clones by TCR betaCDR3 sequencing. Lancet. 2004;364(9431):355-364.

11. de Latour RP, Visconte V, Takaku T, et al. Th17 immune responses contribute to the pathophysiology of aplastic anemia. Blood. 2010;116(20):4175-4184.

12. Solomou EE, Rezvani K, Mielke S, et al. Deficient CD4+ CD25+ FOXP3 $+\mathrm{T}$ regulatory cells in acquired aplastic anemia. Blood. 2007;110(5):1603-1606.

13. Kordasti S, Marsh J, Al-Khan S, et al. Functional characterization of CD4+ T cells in aplastic anemia. Blood. 2012;119(9):2033-2043.

14. Kordasti S, Costantini B, Seidl T, et al. Deep phenotyping of Tregs identifies an immune signature for idiopathic aplastic anemia and predicts response to treatment. Blood. 2016;128(9):1193-1205.

15. Yoshizato T, Dumitriu B, Hosokawa K, et al. Somatic Mutations and Clonal Hematopoiesis in Aplastic Anemia. N Engl J Med. 2015;373(1):35-47.

16. Cooper JN, Young NS. Clonality in context: hematopoietic clones in their marrow environment. Blood. 2017;130(22):2363-2372.

17. Calis JJ, Rosenberg BR. Characterizing immune repertoires by high throughput sequencing: strategies and applications. Trends Immunol. 2014;35(12):581-590.

18. Sallusto F, Geginat J, Lanzavecchia A. Central memory and effector memory $T$ cell subsets: function, generation, and maintenance. Annu Rev Immunol. 2004;22:745-763

19. Hu X, Gu Y, Wang Y, Cong Y, Qu X, Xu C. Increased CD4+ and $\mathrm{CD} 8+$ effector memory $\mathrm{T}$ cells in patients with aplastic anemia. Haematologica. 2009;94(3):428-429.

20. Hosokawa K, Muranski P, Fenx X, et al. Memory Stem T Cells in Autoimmune Disease: High Frequency of Circulating CD8+ Memory Stem Cells in Acquired Aplastic Anemia. J Immunol. 2016;196(4):1568-1578.

\title{
Hematopoietic stem cell mobilization with plerixafor in sickle cell disease
}

\author{
Matthew M. Hsieh and John F. Tisdale
}

\author{
Cellular and Molecular Therapeutics Section, Sickle Cell Branch, National Heart, Lung, and Blood Institute (NHLBI), National \\ Institutes of Health (NIH), Bethesda, Maryland
}

E-mail: matthewhs@nhlbi.nih.gov

doi:10.3324/haematol.2018.190876

A After more than a half-century since the molecular basis for sickle cell disease (SCD) was described by Linus Pauling and colleagues, we now possess the molecular tools to contemplate a one-time cure through genetic modification of autologous hematopoietic stem cells (HSC). For these promising gene transfer and gene editing strategies to become a reality, a sufficient number of HSC of high purity must be obtained. Filgrastim, or granulocyte colony-stimulating factor, mobilization and apheresis is the standard method for HSC collection in healthy adult donors, yet this approach is associated with high rates of adverse events requiring hospitalization in SCD, including vaso-occlusive crises, multi-organ failure, and even death, prompting our call for a moratorium on its use for HSC mobilization in SCD. ${ }^{1}$ Thus, bone marrow harvesting is the default approach, with evidence supporting its utility in both animal models and in vitro studies utilizing patients' material..$^{2-4}$ However, bone marrow harvest- 